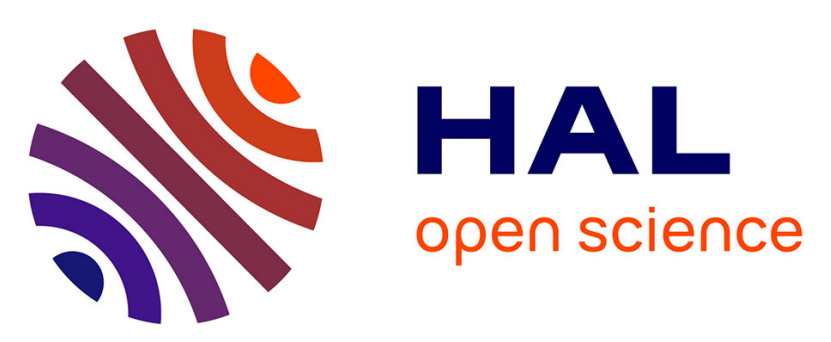

\title{
Rouse 2D diusion of polymer chains in low density precursor films of polybutadiene melts
}

Claire Schune, Marc Yonger, Mohamed Hanafi, Jürgen Thiel, Laurent Guy, Thomas Chaussée, François Lequeux, Hélène Montes, Emilie Verneuil

\section{- To cite this version:}

Claire Schune, Marc Yonger, Mohamed Hanafi, Jürgen Thiel, Laurent Guy, et al.. Rouse 2D diusion of polymer chains in low density precursor films of polybutadiene melts. ACS Macro Letters, In press, pp.843-848. 10.1021/acsmacrolett.0c00144 . hal-02571015v2

\section{HAL Id: hal-02571015 \\ https://hal.science/hal-02571015v2}

Submitted on 27 May 2020

HAL is a multi-disciplinary open access archive for the deposit and dissemination of scientific research documents, whether they are published or not. The documents may come from teaching and research institutions in France or abroad, or from public or private research centers.
L'archive ouverte pluridisciplinaire HAL, est destinée au dépôt et à la diffusion de documents scientifiques de niveau recherche, publiés ou non, émanant des établissements d'enseignement et de recherche français ou étrangers, des laboratoires publics ou privés. 


\title{
Rouse 2D diffusion of polymer chains in low density precursor films of polybutadiene melts
}

Claire Schune, ${ }^{\dagger, \ddagger}$ Marc Yonger, ${ }^{\dagger, \ddagger}$ Mohamed Hanafi, ${ }^{\dagger}$ Jürgen Thiel, $₫$ Laurent Guy, ${ }^{\ddagger}$

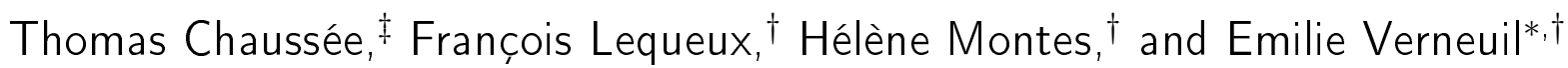

$\dagger$ Soft Matter Sciences and Engineering (SIMM), ESPCI Paris, PSL University, Sorbonne Université, CNRS, F-75005 Paris, France

$\ddagger$ Solvay Silica, 15 rue Pierre Pays, BP 52, F-69660 Collonges-au-Mont-d’Or, France

\Max-Planck Institute for Polymer Research, Ackermannweg 10, D-55128 Mainz, Germany

E-mail: emilie.verneuil@espci.fr

\begin{abstract}
We took advantage of pseudo-partial wetting to promote the spreading of precursor films whose surface density smoothly decays to zero away from a sessile droplet. By following the spreading dynamics of semi-dilute precursor films of polybutadiene melts on silicon wafers, we measure molecular diffusion coefficients for different molar masses and temperatures. For homopolymers, chains follow a thermally activated 2D Rouse diffusion mechanism, with an activation energy revealing polymer segments interactions with the surface. This Rouse model is generalized to chains with specific terminal groups.
\end{abstract}

Describing the dynamics of polymer melts near solid surfaces is of great relevance to mechanics, adhesion, friction or flows at interfaces as they set the boundary conditions and 
the whole dynamic response ${ }^{1-6}$. However, direct information on polymer/substrate interactions can be challenging to obtain. Indeed, the polymer dynamics at interfaces strongly couples polymer/substrate interactions and polymer/polymer interactions in the bulk ${ }^{7}$. In order to isolate the polymer/substrate interactions, we conducted wetting experiments on oxidized silicon wafers to promote the spreading of sub-molecular thickness polymer films, where all the chains are expected to interact with the surface. The surfaces and polymer melts used have a high positive spreading parameter $S=\gamma_{s}-\left(\gamma+\gamma_{s l}\right)$, with $\gamma_{s}$, $\gamma$, and $\gamma_{s l}$ the interfacial tensions of the solid/air, liquid/air and solid/liquid interfaces respectively. In such conditions, past experiments ${ }^{7}$ demonstrated that a so-called precursor film spreads around sessile droplets, driven by short range interactions through the gain in surface energy $S$. Its morphology also depends on the longer range interactions ${ }^{8,9}$. As measured by $\mathrm{X}-$ ray, neutron reflectivity, X-ray diffuse scattering ${ }^{10-13}$, ellipsometry ${ }^{14-20}$, or interferometry ${ }^{21}$, these polymer precursor films are nanometer thick. In a theoretical point of view, restricting to cases where only long range Van der Waals interactions and surface interactions were at play, Brochard-Wyart et al. ${ }^{8}$ distinguished two wetting situations on high energy surfaces, depending on the sign of the Derjaguin pressure $\Pi$ in the film ${ }^{22}$. If the Derjaguin pressure is positive, the long range interactions result in an effective repulsion of the air/polymer and polymer/substrate interfaces, which aims to stabilize the wetting precursor film. This situation is called total wetting ${ }^{23}$ and was widely observed in the past for various polymers ${ }^{10,14-17,17-19,24-30,30-33}$. Such precursor films exhibit a step-like profile. It is expected to be comprised of a near-dense monolayer of molecules, except at the utmost end of the film where the compactness is gradually lost ${ }^{16,17}$. The precise shape and dynamics of the step where chain density decays to zero is believed to contain information on the polymer/surface interactions but the narrowness of this region in the case of total wetting is limiting.

In the present paper we offer to study the wetting of polybutadiene melts on oxidized silicon wafers, which experience conjoining interactions that tend to squeeze the precursor film $(\Pi<0)$ : a motionless droplet coexists with the precursor film. This regime is called 
pseudo-partial wetting and was evidenced for high polarisability polymers ${ }^{8,34-43}$. We will show that depositing polybutadiene droplets on such surfaces is a way to prepare precursor films of low density, which smoothly decay over an extended lateral region. We will characterize the precursor film profiles and dynamics by ellipsometry and show that the chains in the film obey a diffusion equation. The variations of the diffusion coefficient with the film thickness are extracted from the ellipsometry data and its evolution with the molar mass will be tackled for homopolymers and polymers with specific terminal groups. In addition, for homopolymers, the effect of temperature on the diffusion will be studied and translated in terms of activation energy.

The polymer melts used in this study are 1,4-polybutadiene (PBd 1,4), hydroxyl-terminated 1,4-polybutadiene (PBd-OH), and 1,2-polybutadiene (PBd 1,2). Molar masses $M_{n}$ range between 900 and $40000 \mathrm{~g} / \mathrm{mol}^{44,45}$. The glass transition temperature $T_{g}$ of PBd 1,4 and PBd-OH is close to $-100 \mathrm{C}$ and close to $-30 \mathrm{C}$ for $\mathrm{PBd} 1,2$. It does not significantly depend on the molar mass in the range tested ${ }^{44,47}$, which allows to work in the $10 \mathrm{C}$ to $73 \mathrm{C}$ range while being at large temperature distance to $T_{g}$. The precursor films spreading around the droplets were characterized with an ellipsometric microscope (EP3, Accurion) using nulling ellipsometry with $590 \mathrm{~nm}$ wavelength of $10 \mathrm{~nm}$ bandwith, $65^{\circ}$ incidence angle, and five minutes time resolution. Sessile droplets of radius $r_{d}$ comprised between 40 and $250 \mu \mathrm{m}$, and volume below the nanoliter, were deposited on piranha cleaned ${ }^{44}$ oxidized silicon wafers, with an initial oxide layer thickness $e$ measured from 2 to $4 \mathrm{~nm}$ depending on the wafer used. The experiments were performed in an hermetic cell under a weak flow of nitrogen, at a fixed relative humidity of $11 \%$, and at different temperatures. As the silica thickness $e$ is known, the polymer contribution to the optical path shift can be deduced from ellipsometry measurements and the local polymer thickness $h$ can be determined. This thickness is an averaged thickness over the pixel area of the ellipsometric microscope, which is around $64 \mu \mathrm{m}^{2}$. The polymer thickness $h$ may be smaller than a molecular size and in that case the polymer layer is in a non-dense 
state. As demonstrated in literature, the bulk value of the refractive index of polybutadiene still can be used ${ }^{43,48}$. Noise reduction is achieved by taking advantage of the quasi circular shape of the droplets: thicknesses are angularly averaged over $10^{\circ}$ angles centered to the droplet center. Deposition method, experimental set-up, and data analysis were detailed in a previous study ${ }^{43}$. We ensured the volatility of the polymer melts can be neglected and the absence of volatile pollutes by checking that nothing transfers to the surface from a drop kept slightly above it. Atomic force microscopy showed the precursor films have no texture. ${ }^{43}$

The typical spreading of a precursor film of $\mathrm{PBd} 1,4$ around a sessile droplet of radius $r_{d} \simeq 100 \mu \mathrm{m}$ at short times is shown in Fig. 1. A few minutes after the deposition of
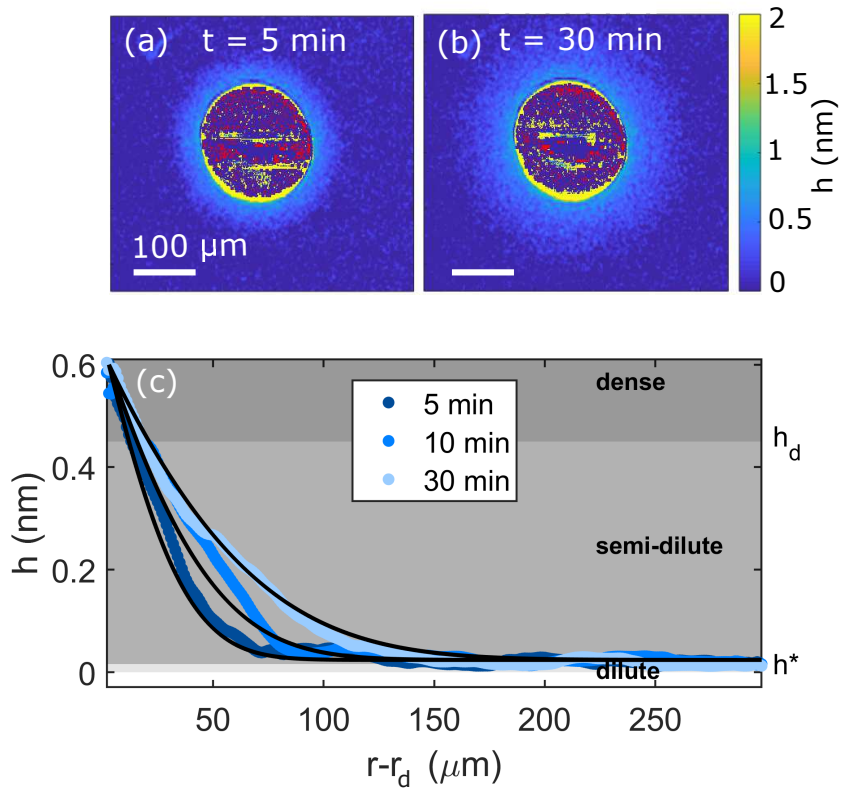

Figure 1: Thickness maps measured by ellipsometry of a precursor film of PBd 1,4 of $M_{n}=$ $1900 \mathrm{~g} / \mathrm{mol}$ at (a) $5 \mathrm{~min}$ and (b) $30 \mathrm{~min}$ after the deposition time of the droplet. (c) Corresponding thickness profiles as a function of the distance to the droplet edge $r-r_{d}$, angularly averaged over a $10^{\circ}$ angle centered at the droplet center. Black lines: fit to solutions of Eq. 1 with $\bar{D}=1.6 \mu \mathrm{m}^{2} / \mathrm{s}$ and $h_{1}=0.6 \mathrm{~nm}$. Silica layer thickness is $e=2.2$ $\mathrm{nm}$ and $T=20 \mathrm{C}$.

the droplet, the contact line stops while a film spontaneously spreads around the droplet. This clearly indicates that PBd exhibits pseudo-partial wetting condition on oxidized silicon 
wafer, which is consistent with the literature $8,40,41,44,49$. As seen in Fig. 1(c), the precursor film is connected to the droplet at a constant nanometric thickness $h_{1}$ and its height profile smoothly decreases from $h_{1}$ towards zero. This constant pinning height at the film/droplet connection is always observed (see SI3 ${ }^{44}$ ) and not correlated to the molar mass. It averages to $h_{1}=0.6 \pm 0.4 \mathrm{~nm}$ for PBd 1,4. Similar precursor film thickness profiles were obtained for all PBd 1,4 $4^{44}, \mathrm{PBd} 1,2^{50}$ and PBd-OH. Remarkably, thickness values stand below both the Kuhn length $b=0.96 \mathrm{~nm}$ and the bulk gyration radius $R_{g}=1.67 \mathrm{~nm}$ for PBd $1900 \mathrm{~g} / \mathrm{mol}^{51}$. It thus raises the question of the polymer density in precursor films, as pointed out in the literature $^{7,10,16,32}$.

Following the path of Johner and Semenov ${ }^{52}$ on confined polymer chains, we derive the surface-averaged thickness in the precursor film, based on topological considerations in two cases: (i) when polymer segments cover the entire silica surface and (ii) at the dilute limit, when the $2 \mathrm{D}$ coils are separated enough so that they do not interact with each other. Chains are considered as cylinders of length $N b$ and diameter $d$, where $N$ is the number of Kuhn segments per polymer chain, so that the molecular volume writes $V_{c}=N b \pi d^{2} / 4$. The diameter $d$ can be estimated by balancing $V_{c}$ with the molecular volume $V=M_{K} N / \rho N_{A}$, where $M_{K}$ is the molar mass of the Kuhn segment, $\rho$ the density of polybutadiene, ${ }^{44}$ and $N_{A}$ the Avogadro constant. We find $d=\sqrt{4 M_{K} /\left(\rho N_{A} b \pi\right)} \simeq 0.53 \mathrm{~nm}$.

In case (i), the surface averaged thickness $h_{d}$ equals the chain volume $V_{c}$ divided by the projected area occupied by a chain on the surface $S_{d}=N b d$ yielding $h_{d}=\frac{\pi}{4} d \sim 0.45 \mathrm{~nm}$ for PBd. When $h>h_{d}$, the film is dense. When $h \leq h_{d}$, some silica sites are unoccupied. In case (ii), many silica sites are unoccupied and the projected area of a chain writes $S^{*}=\pi R_{g, 2 D}^{2}$, $R_{g, 2 D}$ being the $2 \mathrm{D}$ gyration radius. The surface-averaged thickness of a $2 \mathrm{D}$ coil is then $h^{*}=V_{c} / S^{*}$. It is similar to the overlap concentration $c^{*}$ in solution, with the unoccupied silica sites playing the role of the solvent. When $h<h^{*}$, the film is $2 \mathrm{D}$ dilute. Above $h^{*}$, it is $2 \mathrm{D}$ semi-dilute and the chains overlap. For polymer chains confined in $2 \mathrm{D}$ with some overlapping allowed, Johner and Semenov theoretically showed that the variation of $R_{g, 2 D}$ 
with $N$ amounts to a power law with exponent $\nu$ varying with $N$ between $1 / 2$ and $3 / 4$ : $R g_{2 D}=2 b N^{\nu}$. Consequently, thickness $h^{*}$ weakly decreases with $N: h^{*} \sim\left(d^{2} / 16 b\right) N^{1-2 \nu}$. For the mass range of our study, we find $h^{*}<0.03 \mathrm{~nm}$, which is at the detection limit for thicknesses. The precursor film thicknesses $h$ measured by ellipsometry verify $h^{*}<h<h_{d}$ which indicates that the chains in the precursor films are in the 2D semi-dilute regime. Furthermore, at any given time, we observe that, as the distance from the droplet $r-r_{d}$ increases, the thickness $h$ decreases and vanishes at large distance, which we interpret as a decrease in polymer density until the film completely dilutes.

Our observations on $\mathrm{PBd}$ 1,4 show that the pseudo-partial wetting regime drastically differs from what was measured in the literature for polymers in total wetting. In the latter case, the contact line of the droplet continuously advances and the precursor film exhibits a step-like profile of quasi-uniform thickness in the nanometer range ending with a sharp step $^{10,14-18,20,24,25,27-30}$, or in some cases terraced profiles ${ }^{53-56}$. Different models were developed to recover the experimental film profile and time evolution of its lateral extension. In the case of a single step profile, Joanny and de Gennes ${ }^{57}$ derived the thickness profile of the film that they related to a diffusion coefficient in hydrodynamic regime $D(h)=-\left(h^{3} / 3 \eta\right) . d \Pi / d h$. In the case of terraced films with layers of molecular thickness, de Gennes and Cazabat ${ }^{58}$ described the evolution with time of the lateral extension of different layers, but this model only works at sufficiently long times and fails for single layers and/or semi-dilute or dilute layers $^{53,56}$. In a precursor film of vanishing thickness, a hydrodynamic description is not expected to be valid: molecules are dilute and act like a two dimensional gas. In that case, the theoretical diffusion coefficient is constant: $D=k T / \xi$, with $\xi$ the friction coefficient of the molecules on the surface $20,53,54,56,59$.

Here, none of these theoretical frameworks were used a priori. Instead, in order to characterize the spreading dynamics of the polybutadiene chains in our precursor films, the 
experimental data were analyzed with the general diffusion equation (Eq. 1):

$$
\partial_{t} h(r, t)=\frac{1}{r} \partial_{r}\left(r D(h) \partial_{r} h(r, t)\right)
$$

with $r$ the distance to the droplet center. From our experimental profiles, the capillary term described in the literature ${ }^{60-63}$ was found negligible. The variations of the diffusion coefficient $D(h)$ with polymer thickness were recovered by spatially integrating the experimental thickness profiles $h(r, t)$ between two times separated by a time interval $\Delta t^{26,64}$. We chose to consider the diffusion coefficient as an average of the diffusion coefficients measured at $t_{1}=30 \mathrm{~min}$ and with $\Delta t<t_{1}$. This value of $t_{1}$ allows a sufficient lateral extension of the precursor film for all molar masses, so it can be accurately detected. The value of $\Delta t$ is of minor influence. For PBd 1,4, the resulting variations of the diffusion coefficient with the

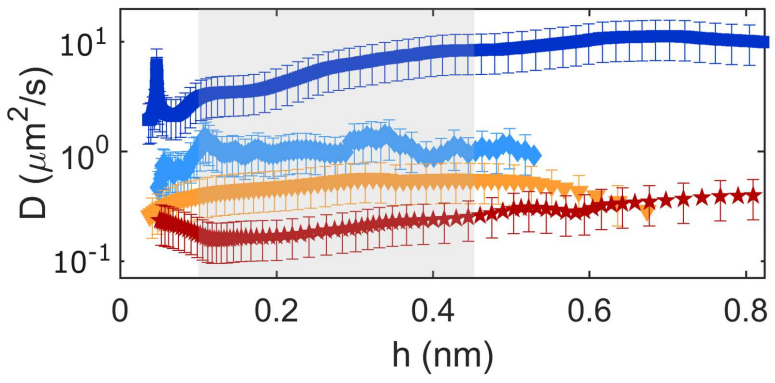

Figure 2: Evolution of the diffusion coefficient $D$ with thickness $h$ for PBd 1,4 at $T=20 \mathrm{C}$. From top to bottom: $M_{n}=900,1900,5400$ and $39100 \mathrm{~g} / \mathrm{mol}$. Grey area: thickness region where $D$ is averaged to obtain $\bar{D}$.

thickness are presented in Fig. 2. Within experimental accuracy, we see that the diffusion coefficient is independent of the polymer thickness for the different molar masses tested. In the following, we define $\bar{D}$ as the average of $D(h)$ in the semi-dilute regime, for $h$ ranging between $0.1 \mathrm{~nm}$ and $h_{d}$. The solution of Eq. 1 with $\bar{D}=1.6 \mu \mathrm{m}^{2} / \mathrm{s}$ and the boundary conditions $h\left(r=r_{d}, t\right)=h_{1}=0.6 \mathrm{~nm}$ and $h\left(r>r_{d}, t=0\right)=0$ for a precursor film of PBd 1,4 $1900 \mathrm{~g} / \mathrm{mol}$ is successfully compared with the experimental data on Fig. 1.

Hence, a thickness independent diffusion coefficient implies the chains dynamics is independent of the polymer surface density even though chains overlap: this suggests chains- 
chains interactions in semi-dilute regime are negligible compared to the chain-surface interactions. From a theoretical point of view, gas lattice models ${ }^{7,65-69}$ and simulations at the microscopic scale ${ }^{64,70-73}$ have been widely used in the literature to describe the growth of molecular films and predict their density profile. Popescu et al. ${ }^{73}$ showed that the film density profile mainly depends on $W_{0}$, the strength of the inter-chains attraction. From this work, our smooth thickness profiles would correspond to $W_{0}<0.4 \mathrm{kT} \simeq 2.5 \mathrm{~kJ} / \mathrm{mol}$. We will see that this value is negligible with respect to the chain/surface interactions we measure.

Figure 3 shows that for $\mathrm{PBd} 1,4$ at 20C, the diffusion coefficient $\bar{D}$ of the polymer chains on the surface is inversely proportional to the chain length. This result holds above the $3 \mathrm{D}$ bulk entanglement length of the polymer $N_{e}^{3 d}=M_{e} / M_{K}=18^{51,74}$. When the temperature is increased to $73 \mathrm{C}$, the power law $\bar{D} \sim N^{-1}$ is maintained and the values of $\bar{D}$ are larger. The PBd chains of the precursor films thus follow a two-dimensional Rouse diffusion mechanism ${ }^{51,75}$, revealing that they do not encounter obstacles or entanglements: in the range of molar masses tested, the chains dynamics in the present $2 \mathrm{D}$ semi-dilute films does not involve any entanglements. This result is in agreement with the recent theory of Lee et $a l .{ }^{76}$, which predicts that the entanglement mass drastically increases with confinement (in $h^{-3}$ ). It also agrees with measurements of the mechanical properties of ultra-thin suspended polymer films. ${ }^{77,78}$

The temperature dependence is tested by fitting $\bar{D}$ to a friction model with an Arrhenius equation $^{26,27}$ :

$$
\bar{D}(N, T)=\frac{k T}{N \xi_{K}}=\frac{k T}{N \xi_{K, \infty} e^{E_{a} / R T}}
$$

where $\xi_{K}$ is the friction coefficient of a polymer segment on the surface, which depends on temperature, friction at infinite temperature $\xi_{K, \infty}$ and activation energy $E_{a}$. The activation energy corresponds to the energy barrier that polymer Kuhn segments have to overcome to move from one site on the surface to another. As seen in Fig. 4(a), by plotting $\bar{D} N /(k T)$ as a function of $1 / T$ for $\mathrm{PBd} 1,4$, all the data collapse on a single master curve, allowing 


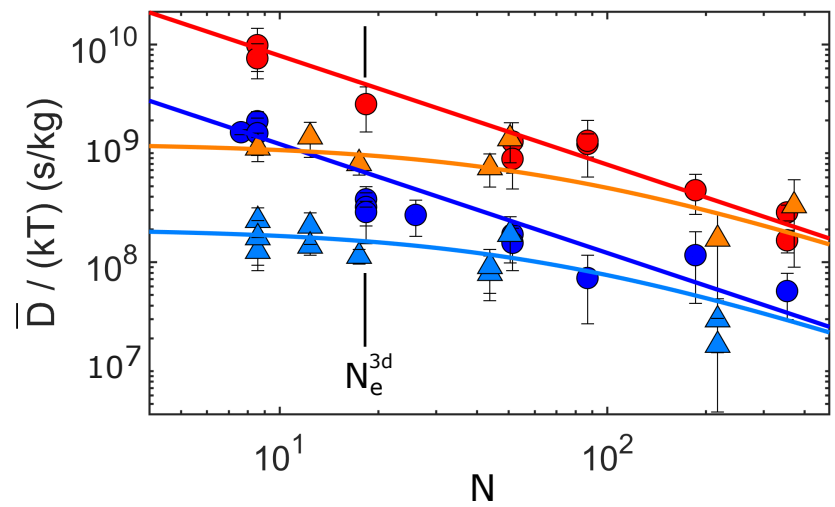

Figure 3: $\bar{D} /(k T)$ as a function of $N$. PBd 1,4: $\bullet$ at $20 \mathrm{C}$, $\bullet$ at $73 \mathrm{C}$. Lines : fit to Eq. 2 with $E_{a}=29.7 \mathrm{~kJ} / \mathrm{mol}$ and $\xi_{K, \infty}=10^{-15.4} \mathrm{~kg} / \mathrm{s}$. PBd-OH: $\triangle$ at $20 \mathrm{C}, \triangle$ at $73 \mathrm{C}$. Lines: fits to Eq. 3 with $\xi_{O H}^{20 C}=10^{-8.3} \mathrm{~kg} / \mathrm{s}$ and $\xi_{O H}^{73 C}=10^{-10.9} \mathrm{~kg} / \mathrm{s}$.

to extract a unique activation energy $E_{a}=30 \pm 5 \mathrm{~kJ} / \mathrm{mol}$. In Fig. $4(\mathrm{~b})$, the power law $N^{-1}$ is found to be robust when plotting $\bar{D} e^{E_{a} / R T}$ versus $N$ for all temperatures. We find $\xi_{K, \infty}=10^{-15.4 \pm 0.9} \mathrm{~kg} / \mathrm{s}$. At 20C, $\xi_{K}=10^{-10.1 \pm 1.8} \mathrm{~kg} / \mathrm{s}$. For PBd 1,4, the molecular diffusion is thus a thermally activated Rouse mechanism. The surface Kuhn segment activation energy $E_{a}$ can be converted into an equivalent spreading parameter $S_{e q}$ by accounting for the surface of a Kuhn segment contacting silica, i.e. $b \times d$. This yields $S_{e q}=E_{a} /\left(N_{A} b d\right)$ and numerically $S_{e q} \simeq 100 \mathrm{~mJ} / \mathrm{m}^{2}$. This high value of $S_{e q}$ is consistent with the existence of a precursor film, which develops in the case of high energy surfaces ${ }^{79}$.

For PBd-OH precursor films, the diffusion coefficient values were extracted at a thickness $h=0.2 \mathrm{~nm}$. As shown in Fig. 3, the variations of $\bar{D}$ with the chain length for PBd 1,4 and PBd-OH overlap at large $N$, but the $N^{-1}$ scaling is lost at small $N$ for PBd-OH. To account for this observation, we note that the friction coefficient of hydroxylated terminal segments is expected to be different from the homopolymer: $\xi_{K} \neq \xi_{O H}$. Assuming additivity of the friction coefficients, Eq. 2 is modified into Eq. 3:

$$
\bar{D}(N, T)=\frac{k T}{(N-1) \xi_{K}+\xi_{O H}}
$$

By fitting the diffusion coefficient values of $\mathrm{PBd}-\mathrm{OH}$ to Eq. 3, we obtain high friction coefficient ratios: $\xi_{O H} / \xi_{K} \sim 65$ at $20 \mathrm{C}$ and $73 \mathrm{C}$. Hydroxylated terminal groups thus ex- 

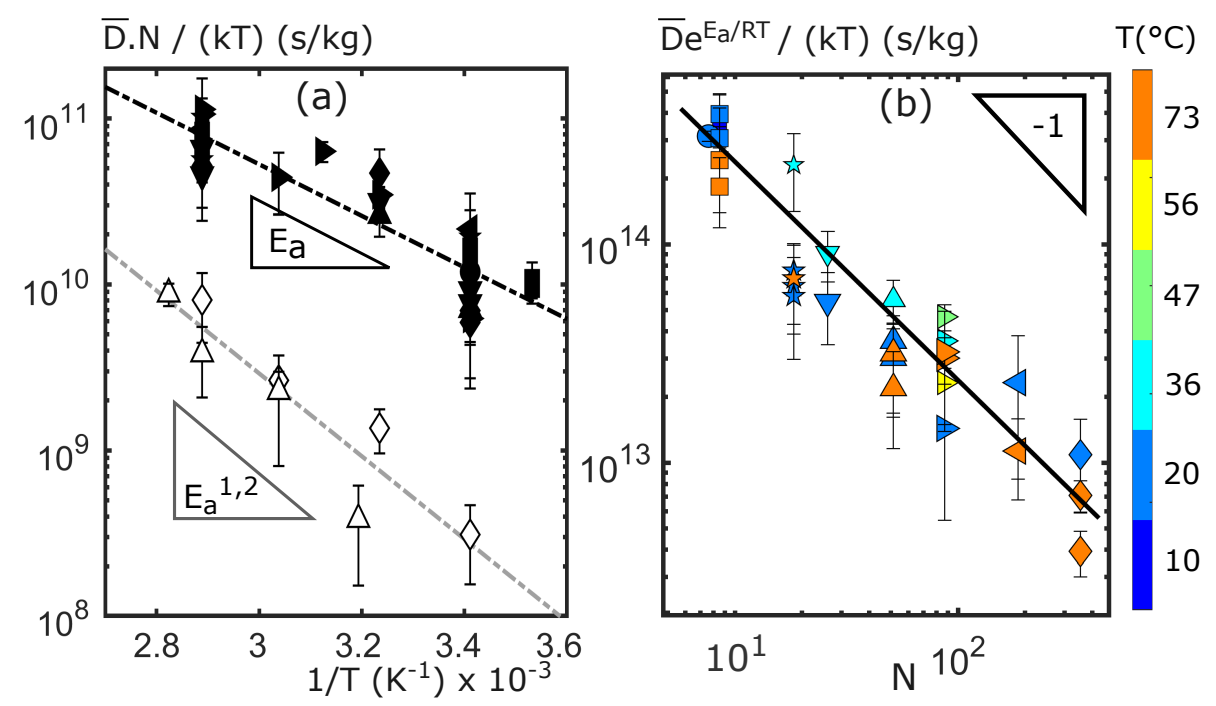

Figure 4: (a) Reduced diffusion coefficient $\bar{D} N /(k T)$ as a function of $1 / T$. The black symbols represent the different PBd 1,4 chain lengths and corresponds to those in (b). Hollow white symbols are for PBd 1,2: $\diamond \mathrm{N}=15, \triangle \mathrm{N}=33$. Dashed lines: fits to Eq. 2: $E_{a}=29.7 \mathrm{~kJ} / \mathrm{mol}$ and $\xi_{K, \infty}=10^{-15.4} \mathrm{~kg} / \mathrm{s}$ for PBd 1,4, and $E_{a}^{1,2}=47.7 \mathrm{~kJ} / \mathrm{mol}$ and $\xi_{K, \infty}^{1,2}=10^{-17} \mathrm{~kg} / \mathrm{s} \mathrm{for}$ PBd 1,2. (b) $\bar{D} e^{E_{a} / R T} /(k T)$ as a function of $N$ allows to collapse all the data for PBd 1,4 on a master curve. Black line: power law with exponent -1 . Color scale: temperature.

perience higher friction on silica. Consequently, the dynamics of a short PBd-OH chain is slowed down compared to a PBd 1,4 chain of equivalent length. For long PBd-OH chains, the Rouse motion of the chains is asymptotically recovered (Fig. 3).

For PBd 1,2 the Rouse scaling of $\bar{D}$ with $N^{-1}$ holds and the molecular diffusion is also thermally activated (Fig. 4). We find a higher activation energy than for PBd 1,4: $E_{a}^{1,2}=48 \pm 5 \mathrm{~kJ} / \mathrm{mol}$, as observed in Fig. $4(\mathrm{a})$. At $20 \mathrm{C}, \xi_{K}^{1,2}=10^{-8.5 \pm 2.9} \mathrm{~kg} / \mathrm{s}$. The value of the surface activation energy thus reveals the specific polymer/substrate interactions, that our measurements allow to quantify. A higher surface activation energy reflects stronger interactions between the homopolymer segments and the substrate, which is the case of PBd 1,2 through its vinyl side groups. ${ }^{80,81}$

In this paper, we show that polybutadiene melts are observed to be in pseudo-partial wetting condition on oxidized silicon. Studying the spreading dynamics of subnanometer thick films allows to probe polymer/interface interactions. We show that the chain density 
in the obtained wetting precursor film decreases with the distance from the droplet: polymer chains are mainly in a 2D semi-dilute state in these films. From the experimental precursor film profiles, we extract the variations of the diffusion coefficient $D$ of the polymer chains on the surface, that we find independent from density. This result suggests little or no interactions in between polymer chains of the precursor films, comparing to polymer/surface interactions. In addition, we find that the diffusion coefficient is inversely proportional to molar mass, which is the signature of a two dimensional Rouse diffusion. This result holds for molar masses below and above bulk entanglement mass, highlighting the non-entangled state in $2 \mathrm{D}$ of the chains in the precursor film. Hence, the Rouse mechanism at stake involves the sole friction of Kuhn segments with the silica interface. By varying the temperature, we are able to determine a surface activation energy on silica for the Kuhn segment of butadiene homopolymers with varied chemistry. A greater surface activation energy was found when vinyl side groups were present, which reveals greater interactions with silica. For hydroxy-terminated polybutadienes, variations of the diffusion coefficient with molar mass were successfully accounted for by modelling the chain friction as the sum of friction coefficients of each of its Kuhn segment.

http://dx.doi.org/10.1021/acsmacrolett.0c00144

This document is the unedited Author's version of a Submitted Work that was subsequently accepted for publication in ACS MacroLetters, copyright (C)American Chemical Society after peer review. To access the final edited and published work see:

http://pubs.acs.org/articlesonrequest/AOR-PHU2RMYUQAWBBYFEJQ59 


\section{Supporting Information Available}

Polymer characterizations, additional experimental specifications, and precursor film thickness profiles. Movie: ellipsometric images for PBD 1,2 Mn=1650 g/mol.

\section{References}

(1) Vogt, B. D. Mechanical and viscoelastic properties of confined amorphous polymers. Journal of Polymer Science, Part B: Polymer Physics 2018, 56, 9-30.

(2) Myshkin, N.; Kovalev, A. Adhesion and surface forces in polymer tribology. A review. Friction 2018, 6, 143-155.

(3) Tsui, O. K.; Russell, T. P.; Hawker, C. J. Effect of interfacial interactions on the glass transition of polymer thin films. Macromolecules 2001, 34, 5535-5539.

(4) Vorvolakos, K.; Chaudhury, M. K. The effects of molecular weight and temperature on the kinetic friction of silicone rubbers. Langmuir 2003, 19, 6778-6787.

(5) Singh, A. K.; Juvekar, V. A. Steady dynamic friction at elastomer-hard solid interface: A model based on population balance of bonds. Soft Matter 2011, 7, 10601-10611.

(6) Bruinsma, R. Slow Spreading of Polymer Melts. Macromolecules 1990, 23, 276-280.

(7) Popescu, M. N.; Oshanin, G.; Dietrich, S.; Cazabat, A. M. Precursor films in wetting phenomena. Journal of Physics Condensed Matter 2012, 24, 1-51.

(8) Brochard-Wyart, F.; di Meglio, J. M.; Quéré, D.; de Gennes, P. G. Spreading of Nonvolatile Liquids in a Continuum Picture. Langmuir 1991, 7, 335-338.

(9) Bonn, D.; Eggers, J.; Indekeu, J.; Meunier, J. Wetting and spreading. Reviews of Modern Physics 2009, 81, 739-805. 
(10) Daillant, J.; Benattar, J. J.; Bosio, L.; Leger, L. Final stages of spreading of polymer droplets on smooth solid surfaces. Epl 1988, 6, 431-436.

(11) Daillant, J.; Benattar, J. J.; Leger, L. Ultrathin films in wetting evidenced by x-ray reflectivity. Physical Review A 1990, 41, 1963-1977.

(12) Daillant, J.; Alba, M. High-resolution x-ray scattering measurements : I . Surfaces. Reports on Progress in Physics 2000, 63, 1725-1777.

(13) Toney, M. F.; Mate, C. M.; Leach, K. A.; Pocker, D. Thickness measurements of thin perfluoropolyether polymer films on silicon and amorphous-hydrogenated carbon with X-ray reflectivity, ESCA and optical ellipsometry. Journal of Colloid and Interface Science 2000, 225, 219-226.

(14) Ausserré, D.; Picard, A. M.; Léger, L. Existence and role of the precursor film in the spreading of polymer liquids. Physical Review Letters 1986, 57, 2671-2674.

(15) Leger, L.; Erman, M.; Guinet-Picard, A. M.; Ausserre, D.; Strazielle, C. Precursor film profiles of spreading liquid drops. Physical Review Letters 1988, 60, 2390-2393.

(16) Heslot, F.; Cazabat, A. M.; Fraysse, N. Diffusion-controlled wetting films. Journal of Physics: Condensed Matter 1989, 1, 5793-5798.

(17) Heslot, F.; Cazabat, A. M.; Levinson, P. Dynamics of wetting of tiny drops: Ellipsometric study of the late stages of spreading. Physical Review Letters 1989, 62, 1286-1289.

(18) Voué, M.; Valignat, M. P.; Oshanin, G.; Cazabat, A. M.; De Coninck, J. Dynamics of Spreading of Liquid Microdroplets on Substrates of Increasing Surface Energies. Langmuir 1998, 14, 5951-5958.

(19) Beaglehole, D. Profiles of the precursor of spreading drops of siloxane oil on glass, fused silica, and mica. Journal of Physical Chemistry 1989, 93, 893-899. 
(20) Cazabat, A. M.; Fraysse, N.; Heslot, F. Thin wetting films. Colloids and Surfaces 1991, 52, $1-8$.

(21) Kavehpour, H. P.; Ovryn, B.; McKinley, G. H. Microscopic and macroscopic structure of the precursor layer in spreading viscous drops. Physical Review Letters 2003, 91, $5-8$.

(22) Derjaguin, B. Definition of the Concept of and Magnitude of the Disjoining Pressure and Its Role in the Statics and Kinetics of Thin Layers of Liquid. Kolloid Zh. 1955, $17,191-197$.

(23) de Gennes, P. G. Wetting: Statics and dynamics. Reviews of Modern Physics 1985, $57,827$.

(24) Villette, S.; Valignat, M. P.; Cazabat, A. M.; Jullien, L.; Tiberg, F. Wetting on the Molecular Scale and the Role of Water. A Case Study of Wetting of Hydrophilic Silica Surfaces. Langmuir 1996, 12, 825-830.

(25) Valignat, M. P.; Fraysse, N.; Cazabat, A. M.; Heslot, F. Molecular Networks in the Spreading of Microdroplets. Langmuir 1993, 9, 601-603.

(26) Novotny, V. J. Migration of liquid polymers on solid surfaces. The Journal of Chemical Physics 1990, 92, 3189-3196.

(27) O’Connor, T. M.; Jhon, M. S.; Bauer, C. L.; Min, B. G.; Yoon, D. Y.; Karis, T. E. Surface diffusion and flow activation energies of perfluoropolyalkylether. Tribology Letters 1995, 1, 219-223.

(28) O’Connor, T. M.; Back, Y. R.; Jhon, M. S.; Min, B. G.; Yoon, D. Y.; Karis, T. E. Surface diffusion of thin perfluoropolyalkylether films. Journal of Applied Physics 1996, 79,5788 . 
(29) Min, B. G.; Choi, J. W.; Brown, H. R.; Yoon, D. Y. Spreading characteristics of thin liquid films of PFPE on solid surfaces. Tribology Letters 1995, 1, 225-232.

(30) Mate, C. M. Anomalous diffusion kinetics of the precursor film that spreads from polymer droplets. Langmuir 2012, 28, 16821-16827.

(31) Ala-Nissila, T.; Herminghaus, S.; Hjelt, T.; Leiderer, P. Diffusive spreading of chainlike molecules on surfaces. Physical Review Letters 1996, 76, 4003-4006.

(32) Albrecht, U.; Achim, O.; Leiderer, P. Two-Dimensional Liquid Polymer Diffusion: Experiment and Simulation. Physical Review Letters 1992, 68, 3192-3196.

(33) Albrecht, U.; Otto, A.; Leiderer, P. Diffusive two-dimensional spreading of a polymer. Surface Science 1993, 283, 383-386.

(34) Silberzan, P.; Léger, L. Evidence for a new spreading regime between partial and total wetting. Physical Review Letters 1991, 66, 185-188.

(35) Esibov, L.; Sarkisov, D.; Jeng, U.-S.; Crow, M. L.; Steyerl, A. Dynamics of pseudopartial wetting studied by neutron reflectometry. Physica B: Condensed matter 1998, 241-243, 1077-1079.

(36) Du, L.; Bodiguel, H.; Cottin, C.; Colin, A. Dynamics of liquid-liquid menisci in pseudopartial wetting. Chemical Engineering and Processing: Process Intensification 2013, $68,3-6$.

(37) Moon, J.; Garoff, S.; Wynblatt, P.; Suter, R. Pseudopartial Wetting and Precursor Film Growth in Immiscible Metal Systems. Langmuir 2004, 20, 402-408.

(38) Reiter, G. Unstable Thin Polymer Films: Rupture and Dewetting Processes. Langmuir 1993, 9, 1344-1351. 
(39) Müller-Buschaum, P.; Vanhoorne, P.; Scheumann, V.; Stamm, M. Observation of nanodewetting structures Observation of nano-dewetting structures. Europhysics Letters 1997, 40, 655-660.

(40) Seemann, R.; Herminghaus, S.; Jacobs, K. Dewetting patterns and molecular forces: A reconciliation. Physical Review Letters 2001, 86, 5534-5537.

(41) Seemann, R.; Herminghaus, S.; Jacobs, K. Gaining control of pattern formation of dewetting liquid films. Journal of Physics Condensed Matter 2001, 13, 4925-4938.

(42) Müller-Buschbaum, P.; Bauer, E.; Wunnicke, O.; Stamm, M. The control of thin film morphology by the interplay of dewetting, phase separation and microphase separation. Journal of Physics Condensed Matter 2005, 17.

(43) Schune, C.; Yonger, M.; Bresson, B.; Fretigny, C.; Guy, L.; Chaussée, T.; Lequeux, F.; Montes, H.; Verneuil, E. Combining Ellipsometry and AFM to Probe Subnanometric Precursor Film Dynamics of Polystyrene Melts. Langmuir 2019, 35, 7727-7734.

(44) See Supplemental Material at [URL will be inserted by publisher].

(45) Liu, C.; Bonaccurso, E.; Sokuler, M.; Günter, K. A.; Butt, H. J. Dynamic wetting of polyisoprene melts: Influence of the end group. Langmuir 2010, 26, 2544-2549.

(46) Pottier, B.; Ducouret, G.; Frétigny, C.; Lequeux, F.; Talini, L. High bandwidth linear viscoelastic properties of complex fluids from the measurement of their free surface fluctuations. Soft Matter 2011, 7, 7843-7850.

(47) Colby, R. H.; Fetters, L. J.; Graessley, W. W. Melt Viscosity-Molecular Weight Relationship for Linear Polymers. Macromolecules 1987, 20, 2226-2237.

(48) Lekner, J. Second-order ellipsometric, coefficients. Physica A 1982, 506-520.

(49) Israelachvili, J. N. Intermolecular and Surface Forces, academic ed.; 1992. 
(50) See Supplemental Material at [URL will be inserted by publisher].

(51) Rubinstein, M.; Colby, R. H. Polymer Physics; New York: Oxford University Press, 2003.

(52) Semenov, A. N.; Johner, A. Theoretical notes on dense polymers in two dimensions. European Physical Journal E 2004, 480, 469-480.

(53) Fraysse, N.; Valignat, M. P.; Cazabat, A. M.; Heslot, F.; Levinson, P. The Spreading of Layered Microdroplets. 1993.

(54) Valignat, M. P.; Oshanin, G.; Villette, S.; Cazabat, A. M.; Moreau, M. Molecular weight dependence of spreading rates of ultrathin polymeric films. Physical Review Letters 1998, 80, 5377-5380.

(55) Heslot, F.; Cazabat, A.; Fraysse, N.; Levinson., P. Experiments on spreading droplets and thin films. Advances in Colloid and Interface Science 1992, 39, 129-145.

(56) Valignat, M. P.; Fraysse, N.; Levinson, P.; Heslot, F.; Cazabat, A. M. Some specific aspects of the behaviour of ultrathin films. Colloids and Surfaces A: Physicochemical and Engineering Aspects 1994, 83, 193-197.

(57) Joanny, J.; de Gennes, P.-G. Upward creep of a wetting fluid : a scaling analysis. Journal de Physique 1986, 47, 121-127.

(58) de Gennes, P. G.; Cazabat, A. M. Etalement d'une goutte stratifiée incompressible. $C$. R. Acad. Sc. Paris 1990, 301, 1601-1606.

(59) Cazabat, A. M.; Fraysse, N.; Heslot, F.; Carles, P. Spreading at the microscopic scale. Journal of Physical Chemistry 1990, 94, 7581-7585.

(60) Brochard, F.; de Gennes, P. G. Spreading laws for liquid polymer droplets. Interpretation of the foot. J. Physique Lett. 1984, 45, 597-602. 
(61) Churaev, N. V.; Starov, V. M. The Shape of the Transition Zone between a Thin Film and Bulk Liquid and the Line Tension. Journal of Colloid and Interface Science 1982, 89.

(62) Benzaquen, M.; Fowler, P.; Jubin, L.; Salez, T.; Dalnoki-Veress, K.; Raphaël, E. Soft Matter. Soft Matter 2014, 10, 8608-8614.

(63) Cormier, S. L.; McGraw, J. D.; Salez, T.; Raphaël, E.; Dalnoki-Veress, K. Beyond tanner's law: Crossover between spreading regimes of a viscous droplet on an identical film. Physical Review Letters 2012, 109, 1-5.

(64) Moon, J.; Yoon, J.; Wynblatt, P.; Garoff, S.; Suter, R. M. Simulation of spreading of precursing Ag films on $\mathrm{Ni}\left(\begin{array}{l}1 \\ 0\end{array}\right)$. Computational Materials Science 2002, 25, 503-509.

(65) Burlatsky, S. F.; Oshanin, G.; Cazabat, A. M.; Moreau, M.; Reinhardt, W. P. Spreading of a thin wetting film: Microscopic approach. Physical Review E 1996, 54, 3832-3845.

(66) Burlatsky, S. F.; Oshanin, G.; Cazabat, A. M.; Moreau, M. Microscopic model of upward creep of an ultrathin wetting film. Physical Review Letters 1996, 76, 86-89.

(67) Burlatsky, S. F.; Cazabat, A. M.; Moreau, M.; Oshanin, G.; Villette, S. Instabilities and Nonequilibrium Structures VI. Springer, Dordrecht; 2000; pp 233-267.

(68) Oshanin, G.; De Coninck, J.; Cazabat, A. M.; Moreau, M. Dewetting, partial wetting, and spreading of a two-dimensional monolayer on solid surface. Physical Review E 1998, 58, R20-R23.

(69) Oshanin, G.; De Coninck, J.; Cazabat, A. M.; Moreau, M. Microscopic model for spreading of a two-dimensional monolayer. Journal of Molecular Liquids 1998, 76 , $195-219$. 
(70) D’Ortona, U.; De Coninck, J.; Koplik, J.; Banavar, J. R. Terraced spreading mechanisms for chain molecules. Physical Review E - Statistical Physics, Plasmas, Fluids, and Related Interdisciplinary Topics 1996, 53, 562-569.

(71) Haataja, M.; Nieminen, J. A.; Ala-Nissila, T. Dynamics of the spreading of chainlike molecules with asymmetric surface interactions. Physical Review E 1996, 53, 51115122.

(72) Isele-Holder, R. E.; Ismail, A. E. Classification of precursors in nanoscale droplets. Physical Review E 2016, 93, 1-8.

(73) Popescu, M. N.; Dietrich, S. Model for spreading of liquid monolayers. Physical Review E 2004, 69, 19.

(74) Fetters, L. J.; Lohse, D. J.; Graessley, W. W. Chain Dimensions and Entanglement Spacings in Dense Macromolecular Systems. Journal of Polymer Science Part B: Polymer Physics 1998, 1023-1033.

(75) Rouse, P. E. A theory of the linear viscoelastic properties of dilute solutions of coiling polymers. The Journal of Chemical Physics 1953, 21, 1272-1280.

(76) Lee, N.-K.; Diddens, D.; Meyer, H.; Johner, A. Local Chain Segregation and Entanglements in a Confined Polymer Melt. Physical Review Letters 2017, 067802, 1-5.

(77) Bodiguel, H.; Fretigny, C. Reduced Viscosity in Thin Polymer Films. Physical Review Letters 2007, 97, 266105.

(78) Si, L.; Massa, M. V.; Dalnoki-Veress, K.; Brown, H. R.; Jones, R. A. L. Chain Entanglement in Thin Freestanding Polymer Films. Physical Review Letters 2005, 127801, $1-4$.

(79) De Gennes, P.-G.; Brochard-Wyart, F.; Quéré, D. Gouttes, bulles, perles et ondes, belin ed.; 2005. 
(80) Choi, S.-S.; Kim, I.-S. Filler polymer interactions in filled polybutadiene compounds. European Polymer Journal 2002, 38, 1265-1269.

(81) Legrand, A. P.; Lecomte, N.; Vidal, A.; Haidar, B.; Papirer, E. Application of NMR Spectroscopy to the Characterization of Elastomer / Filler Interactions. Journal of Applied Polymer Science 199, 46, 2223-2232.

(82) Bandrup, J.; Immergut, E.; Grulke, E. A. Polymer Handbook 4th Ed; John Wiley and Sons.

(83) Green, M. A.; Keevers, M. J. Optical properties of intrinsic silicon at 300 K. Progress in Photovoltaics 1995, 3, 189-192. 
Graphical TOC Entry

$\prod$ 